\title{
Cordonnier Grade 3 Parasitic Complication, Central Nervous System or Other Organ Toxoplasmosis
}

National Cancer Institute

\section{Source}

National Cancer Institute. Cordonnier Grade 3 Parasitic Complication, Central Nervous

System or Other Organ Toxoplasmosis. NCI Thesaurus. Code C138313.

Any Toxoplasmosis infection with central nervous system involvement or other organ involvement. 